\title{
Measuring Ion Oscillations at the Quantum Level with Fluorescence Light
}

\author{
G. Cerchiari® ${ }^{1, *}$ G. Araneda $\odot,{ }^{1,2}$ L. Podhora $\odot,{ }^{3}$ L. Slodička, ${ }^{3}$ Y. Colombe, ${ }^{1}$ and R. Blatt $\odot^{1,4}$ \\ ${ }^{1}$ Institut für Experimentalphysik, Universität Innsbruck, Technikerstrasse 25, 6020 Innsbruck, Austria \\ ${ }^{2}$ Department of Physics, University of Oxford, Clarendon Laboratory, Parks Road, Oxford OX1 3PU, United Kingdom \\ ${ }^{3}$ Department of Optics, Palacký University, 17. Listopadu 12, 77146 Olomouc, Czech Republic \\ ${ }^{4}$ Institut für Quantenoptik und Quanteninformation, Österreichische Akademie der Wissenschaften, \\ Technikerstrasse 21a, 6020 Innsbruck, Austria
}

(Received 7 October 2020; accepted 17 May 2021; published 4 August 2021)

\begin{abstract}
We demonstrate an optical method for detecting the mechanical oscillations of an atom with singlephonon sensitivity. The measurement signal results from the interference between the light scattered by a trapped atomic ion and that of its mirror image. We detect the oscillations of the atom in the Doppler cooling limit and reconstruct average trajectories in phase space. We demonstrate single-phonon sensitivity near the ground state of motion after electronically induced transparency cooling. These results could be applied for motion detection of other light scatterers of fundamental interest, such as trapped nanoparticles.
\end{abstract}

DOI: 10.1103/PhysRevLett.127.063603

Observing trapped ion oscillations is an established research technique for precision measurements of fundamental constants and for studying fundamental quantum physics [1-3]. In the trap, ions are confined in free space and are well isolated from the surrounding environment. Observation of their oscillations around the equilibrium position allows, for example, the precise determination of the masses of atomic elements and of the electron [4,5], as well as testing the symmetries between matter and antimatter [6,7]. Motional quantum effects can also be explored and manifest themselves at the lowest temperatures of the oscillator [8-10]. In trapped ions, cooling and detection of nonclassical states of motion are achieved via laser interaction $[11,12]$. These states are observed by reconstructing the Wigner function by quantum state tomography [13] or by measuring phonon state populations [14]. In such experiments, sensitivity at the quantum level relies on the electronic structure of the ion, which allows resolving and addressing the motional sidebands in one of its transitions [15].

In systems larger than ions, such as mesoscopic micromembranes, microcantilevers, and levitated nanoparticles, the measurement of oscillations offers interesting prospects for force sensing and fundamental investigations in the quantum regime [16,17]. Unlike atoms, these systems do not possess narrow internal transitions, but they can be cooled to the motional ground state [18-20]. As for

Published by the American Physical Society under the terms of the Creative Commons Attribution 4.0 International license. Further distribution of this work must maintain attribution to the author(s) and the published article's title, journal citation, and DOI. atomic ions, levitated nanoparticles are trapped in free space. For nanoparticles, the lowest temperatures are achieved via coherent light scattering in a Fabry-Pérot cavity [21-23]. Another approach is the direct observation of the particle position via light scattering for active feedback cooling [24]. In this regime, resolving a single quanta of motion may allow feedback cooling to the ground state and the observation of quantum states of motion.

Experiments with atomic ions have used the light scattered on a dipole transition for motion analysis and feedback cooling [25-27]. In this context, it was shown that the variance $\left\langle q^{2}\right\rangle$ of an ion state of motion could be resolved with the precision of a single quantum [28]. The signal was obtained by averaging the detected light over several oscillations of the ion. This method cannot be applied directly to characterize a quantum state of motion because the constant and oscillating parts of $\left\langle q^{2}\right\rangle$ are not resolved separately. Sensing the motion of a trapped ion at the single-quantum level in a time-dependent fashion is still a challenging task. However, improved detection schemes of the scattered radiation can allow for the direct analysis of a trapped particle's quantum motion. Recently, it has been predicted that the light scattered by a dipole emitter could grant such sensitivity for the position operator $\langle q\rangle$ [29]. Thus, feedback cooling of a nanoparticle to its ground state of motion should be possible without the need for an optical cavity. Similarly, measurement of atomic motion at the quantum level may be accessible without the need for sideband spectroscopy on a narrow transition. In this Letter, we demonstrate that self-interference of light scattered by a trapped ion can provide sensitivity at the singlephonon level for the ion's oscillations. First, we show simultaneous detection of the ion's oscillation modes and the reconstruction of phase-space trajectories. Then, we 
analyze the technique's sensitivity near the motional ground state.

We confine a single ${ }^{138} \mathrm{Ba}^{+}$ion in a linear Paul trap. The motion of the ion has three orthogonal modes of oscillation with frequencies $\omega_{x} / 2 \pi \sim 1.61 \mathrm{MHz}, \omega_{y} / 2 \pi \sim 1.65 \mathrm{MHz}$, and $\omega_{z} / 2 \pi \sim 0.92 \mathrm{MHz}$. The radio frequency field used for radial confinement is driven at $\Omega_{\mathrm{rf}} / 2 \pi \sim 15.1 \mathrm{MHz}$. The ion is Doppler cooled on the $6 \mathrm{~S}_{1 / 2} \leftrightarrow 6 \mathrm{P}_{1 / 2}$ transition at $493 \mathrm{~nm}$ with a laser beam propagating in a direction with projection over all three normal modes [30]. The motion of the ion is studied by detecting the fluorescence light at $493 \mathrm{~nm}$ emitted by the ion in the interferometric configuration depicted in Fig. 1(a). The fluorescence light is imaged by two in-vacuum confocal objectives with NA = 0.40 [31]. The light collected by one of the objectives is reflected by a distant mirror ( $\sim 30 \mathrm{~cm}$ away from the ion) back to the ion. The reflection is superimposed to the direct fluorescence through the other objective. The photons collimated by the second objective are directed to two single-mode fiber avalanche photodiode detectors using a

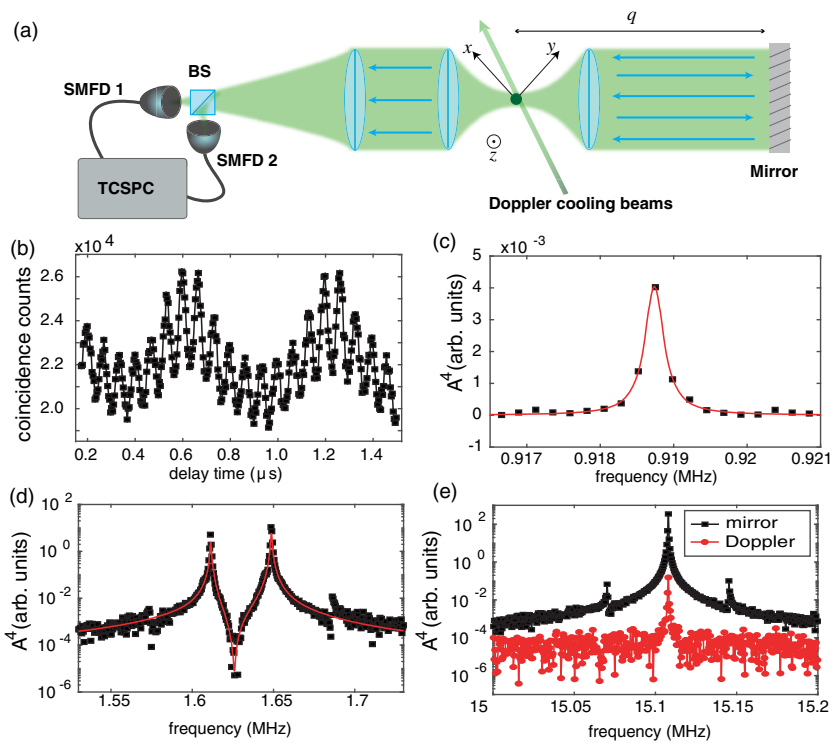

FIG. 1. Detection of the ion motion by self-interference of the light emitted during continuous Doppler cooling. (a) Optical setup. The Doppler beam has an overlap with all the oscillatory modes, whereas the optical detection axis has an overlap only with the radial modes ( $x-y$ plane). The ion's fluorescence is backreflected by a mirror and detected by two single-mode fiber detectors and time tagged with a two-channel time-correlated single photon counting system. (b) Autocorrelation histogram of the photon detection events. Micromotion is visible, superimposed to a slower secular oscillation in the radial modes ( $x$ and $y$ axes). The line is meant to guide the eye. (c)-(e) Power spectra of the autocorrelation. (c) Oscillation on the axial mode ( $z$ axis), orthogonal to the optical axis. (d) Radial modes. The red curves in (c),(d) are fit functions to the data. (e) Micromotion. The black data corresponds to the setup (a), while the red data are taken while blocking the reflection of the mirror. The lines are meant to guide the eye. beam splitter. The arrival of each photon is time tagged with a two-channel time-correlated single photon counting system (PicoHarp 300) with 4 ps resolution. The experiments are limited both by the dead time (100 ns) and by the jitter (0.8 ns) of each avalanche photodiode.

The photon count rate $R$ results from the interference of the primary and reflected light beams. The measured rate depends on the mutual distance $q$ between the ion and the mirror through

$$
R=R_{0}\left[1+\mathcal{V} \sin \left(\frac{4 \pi}{\lambda} q\right)\right]
$$

where $\mathcal{V}$ is the contrast of the interference and $R_{0}$ is the average photon count rate. If the position of the mirror is kept fixed, $q$ depends only on the ion oscillations resulting in a modulation of the photon count rate $R$. Real-time measurement of $q$ was demonstrated in Ref. [25]. The scheme required narrow-bandwidth amplification by mixing the photon stream with a local oscillator signal. The interference of scattered light is already used for the detection of nanoparticle motion [24]. However, the interference is typically achieved homodyning the focused Gaussian beam illuminating the nanoparticle with the scattered dipole field. Our scheme based on self-interference is more flexible by allowing the orientation of the detection at an arbitrary angle. Moreover, we collect the position information contained in both the primary and reflected fluorescence light that interfere on the detector. These two features can be used to realize an ideal theoretical arrangement [29] that should provide detection at the Heisenberg limit (see D in the Supplemental Material [32]).

In this work, photon arrival times are recorded and postprocessed. Time tagging allows the detection of all the modes simultaneously without band filtering. Motion detection using photon time tagging and postprocessing was demonstrated in Ref. [33], where, however, the photon count rate was solely modulated by the Doppler effect without the use of optical interference.

Continuous Doppler cooling is applied by the $493 \mathrm{~nm}$ beam detuned by $\Delta / 2 \pi=-35 \mathrm{MHz}$ and with a Rabi frequency $\Omega / 2 \pi \sim 12 \mathrm{MHz}$. The repumper at $650 \mathrm{~nm}$ is detuned by $\Delta_{r} / 2 \pi=-10 \mathrm{MHz}$ and induces a Rabi frequency of $\Omega_{r} / 2 \pi \sim 14.2 \mathrm{MHz}$. These values are estimated by fitting Bloch equation steady-state solutions to scans of the fluorescence intensity [34]. In this condition, a visibility of $\mathcal{V} \sim 33 \%-37 \%$ is typically measured by scanning the mirror position over an interference fringe with an average detected photon count rate of $(5-6) \times 10^{4} \mathrm{~s}^{-1}$. To detect the motion, photon events are registered for $\sim 900 \mathrm{~s}$ under steady-state Doppler cooling of the trapped ion. Figure 1 presents the analysis of data acquired in a single experimental run. The ion motion is studied by computing the autocorrelation of the photon events. A section of the autocorrelation is shown in Fig. 1(b), where both secular 
and micromotion oscillations are visible. Figures 1(c)-(e) show the frequency peaks present in the power spectrum of the autocorrelation. Since the autocorrelation function is the Fourier transform of the power spectrum, further calculating the power spectrum of the autocorrelation leads to a spectral signal proportional to the fourth power of the excursion amplitude $A$ (see scales in the Fig. 1). The peak in Fig. 1(c) corresponds to the axial (z-axis) mode, which has the smallest overlap with the optical axis of the confocal objectives. Figure 1(d) shows the $\mathrm{x}$ and $\mathrm{y}$ secular modes.

The system can be modeled assuming that the ion evolves following free trajectories of oscillation on the three normal modes randomly perturbed at discrete times. Such events are generated by photon absorption and emission, collisions with the background gas, or perturbation in the trapping electromagnetic field. All these events modify the oscillator phase with a random reinitialization but affect all the modes simultaneously. We used these assumptions to calculate suitable fit functions [red curves in Figs. 1(c),(d)] for our data in linear expansion of Eq. (1) in $q / \lambda$. Our model describes the data for 5 orders of magnitude near the oscillation peaks of the radial modes ( $\mathrm{x}$ and $\mathrm{y}$ ), also identifying the interference minimum that appears at $\sim 1.625 \mathrm{MHz}$ between the modes [see Fig. 1(d)]. The minimum arises from the analysis of the motion via the two-photon correlation function, which fixes the phases of oscillations of the different modes to zero at correlation time $t=0$.

Further nonlinear terms in the expansion series of Eq. (1) contribute with additional peaks in the spectrum. Thanks to the nonlinear peaks at $2 \omega_{x}-\omega_{y} \sim 1.57 \mathrm{MHz}$ and $2 \omega_{y}-\omega_{x} \sim 1.69 \mathrm{MHz}$, we estimate that the ion oscillates with 115-125 nm excursion in the radial modes (peak to peak). More details about the model function and the amplitude estimation can be found in B in the Supplemental Material [32]. Figure 1(e) shows the micromotion peak. Micromotion can also be detected without the reflection from the mirror (red data points) thanks to the Doppler effect [35]. However, our measurement shows that the selfinterference technique (black data points) delivers a five to sevenfold improvement in amplitude sensitivity [36]. We believe that the measured residual micromotion can be largely attributed to phase differences in the rf driving applied to the electrodes due to imperfections in the wiring of the trap [37].

Time tagging can also be used to reconstruct phase-space trajectories in reference to a state preparation. Similar to other schemes [38,39], we impress a phase to the ion oscillation by an external drive. The position and phase of the harmonic motion are obtained by synchronizing time tagging with the drive phase and by averaging over several realizations. Under Doppler cooling conditions, we drive the y mode for $50 \mu \mathrm{s}$, which results in $100 \mathrm{~nm}$ excursion oscillation with a well-defined phase. The experiment is
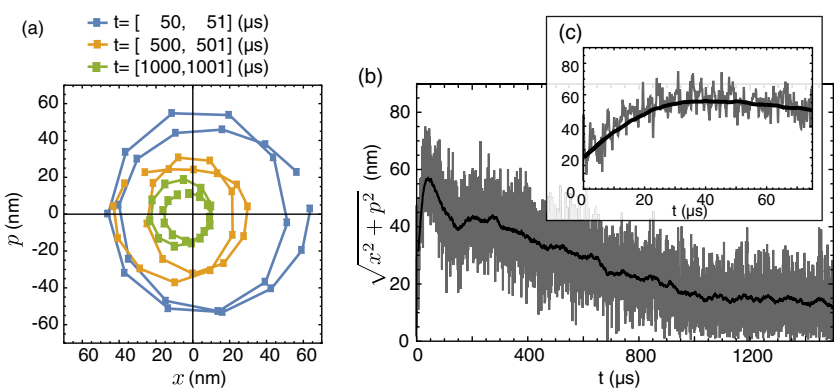

FIG. 2. Ion motion after an initial coherent excitation. (a) Average phase-space trajectory in three $1 \mu \mathrm{s}$ time windows at $t=\{50,500,1000\} \mu \mathrm{s}$. The slight asymmetry of the trajectories is caused by shot noise in the measured oscillation amplitude (see $\mathrm{C}$ in the Supplemental Material [32]). Error bars ( $\sim 8 \mathrm{~nm}$ in both quadratures) are omitted for readability. (b) Phase-space amplitude of the coherent oscillation. The gray line shows the original data; the black line is an $8 \mu$ s moving average. The oscillation profile is displayed up to $1.4 \mathrm{~ms}$, including the rf-driven part and ringdown. (c) An enlargement of the first $80 \mu \mathrm{s}$.

repeated $\sim 5 \times 10^{5}$ times. The starting point of the rf drive is used for correlation analysis to calculate the histogram of photon events arriving at specific time delays from the drive start. The histogram is filtered and analyzed in Fourier space to reconstruct the experimental trajectories of the position $q$ and momentum $p$ quadratures (see $\mathrm{C} 1$ in the Supplemental Material [32]). The $p$ quadrature is normalized by a factor $1 / m \omega_{y}$, where $m$ is the mass of the ion. The combined position and momentum data are plotted in phase space in Fig. 2(a) for different time intervals, showing features of a coherent state with decaying amplitude. Figure 2(b) shows the amplitude of the oscillation calculated as the distance from the center of the phase space. An exponential decay fit to the $q$ quadrature gives a $\tau=$ $847(12) \mu$ s time constant. The coherence time is unchanged when using a pulsed, instead of continuous, illumination, ruling out an effect of the cooling laser. Further measurements by sideband spectroscopy indicate a trap heating rate $<10$ phonon/s (see below). Thus, we concluded that the observed decay is due to progressive dephasing of the ion oscillations caused by instability in the rf trap drive and that it emerges from averaging over multiple runs (see A2 in the Supplemental Material [32]).

To study the sensitivity of this method near the ground state of motion, the radial modes of a single ion are cooled using electromagnetically induced transparency (EIT) (see A1 in the Supplemental Material [32]) [40]. The average phonon number $\langle n\rangle$ is measured by sideband spectroscopy on the $6 \mathrm{~S}_{1 / 2} \rightarrow 5 \mathrm{D}_{5 / 2}$ transition at $1.76 \mu \mathrm{m}$ by comparing the strengths of the red and blue sidebands [15]. We vary the length of the EIT cooling pulse between 0.1 to $5 \mathrm{~ms}$ to obtain $\langle n\rangle$ between 7 and 0.5. After EIT cooling, the motion of the ion is probed by using the Doppler beam for $120 \mu \mathrm{s}$. We note that the light scattered from the Doppler beam also perturbs the state by back action. With the beam 
on, the heating rate of the ion ground state of motion increases from < 10 phonons/s to 1200 phonons/s as measured by sideband spectroscopy.

The sequence consisting of Doppler cooling, EIT cooling, and pulsed Doppler illumination is repeated $\sim 6 \times 10^{5}$ times over $900 \mathrm{~s}$, during which the setup is sufficiently stable (see A3 in the Supplemental Material [32]). Figure 3 shows detection of the ion motion at a low phonon number using the autocorrelation signal of the photons scattered from the Doppler beam. The spectral power at $\omega_{y}$ as a function of the EIT cooling time is given in Fig. 3(a). Here, the statistical uncertainties of the spectral powers are estimated from the background in the spectrum. The blue curve in the main figure is the theoretical squared expectation value of the $\langle q\rangle$ operator for coherent states having a mean number of phonon number $\langle n\rangle$. In this model, $\langle q\rangle^{2}=\cos ^{2}(\theta) \hbar\langle n\rangle / m \omega_{y}$, where $\theta=37^{\circ}$ is the projection angle between the $y$ axis and the optical axis (see B1 in the Supplemental Material [32]). The coherent state model was selected because the method is measuring the amplitudes of coherent oscillations detected at specific frequencies and no amplitude of oscillations should be measurable for the ground state $(\langle n\rangle=0)$. The measured points are offset and scaled in the vertical axis to calibrate the amplitude such that the point at $\langle n\rangle=2$ corresponds to the theoretical value. This data allow us to prepare the initial phonon numbers $\langle n\rangle=2.0(5), 1.0(3)$, and 0.5(2) with suitable EIT
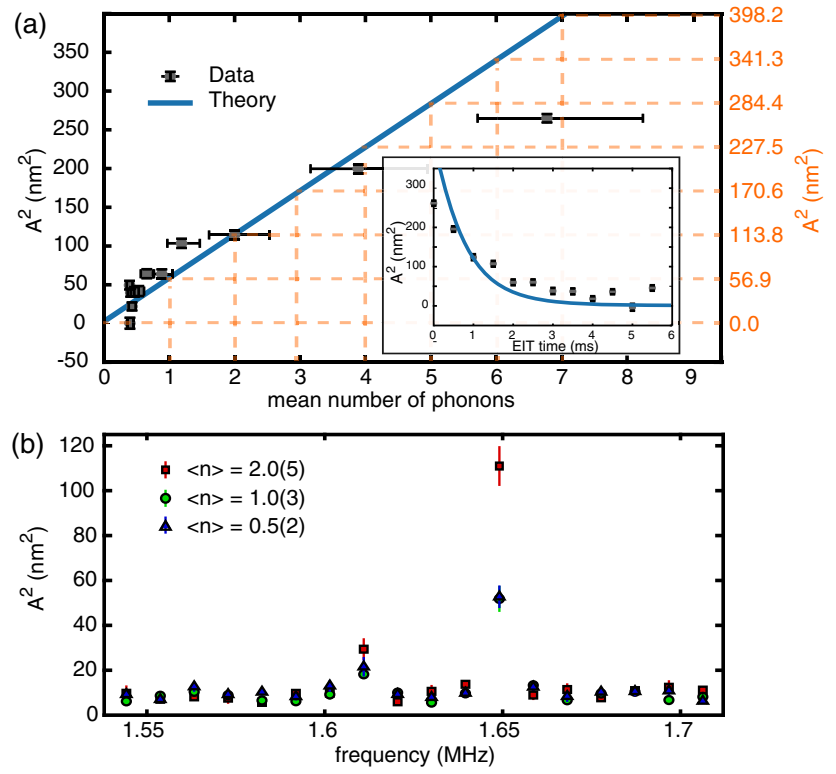

FIG. 3. (a) Power spectral density of the $\omega_{y} / 2 \pi=1.65 \mathrm{MHz}$ mode as a function of the number of phonons (main figure) and electromagnetically induced transparency (EIT) cooling time (inset). The orange grid shows the theoretical values for Fock states. The blue lines correspond to the square of the theoretical expectation value of the $q$ operator at the direction of detection for a coherent state (see main text). (b) Power spectrum of the radial ion motion for initial phonon numbers 2.0(5), 1.0(3), and 0.5(2). pulse lengths and compare the sensitivity of the two techniques. An alternative calibration technique that does not resort to sideband spectroscopy is introduced in B2 of the Supplemental Material [32]. For the selected $\langle n\rangle$, the measurement is repeated 5 times. These acquisitions are used to compute the power spectra of Fig. 3(b) evaluated from the photon autocorrelation. Considering the $\omega_{y}$ mode selected for calibration, the two-phonon and one-phonon spectral powers are distinguished by a $5.6 \sigma$ deviation. At phonon numbers 1.0(3) and 0.5(2), our analysis indicates that the two measurements are compatible $(<1 \sigma)$. We attribute this to instabilities in the interference fringe offset over the total measuring time of several hours (see A3 in the Supplemental Material). The measurements presented in Fig. 3 show how our method can be applied to study the cooling and heating dynamics of diverse mechanical oscillators for multiple motional modes simultaneously.

In this Letter, we have demonstrated that the stream of single photons emitted by a trapped ion can be analyzed to detect its motion at the single quantum level. We have presented direct optical detection of the ion's mechanical oscillations and measured their amplitude, frequency, and phase. The presented methods do not rely on the internal electronic structure of the ion and, thus, may find application for other oscillators such as levitated nanoparticles. The technique is limited by the stability of the interference fringes (see A3 in the Supplemental Material [32]) and, ultimately, by the back action of the scattered light. By adopting higher NA optics, the signal over the backaction effect can be improved to realize either faster or more precise acquisitions. This hypothesis can be tested in already existing setups featuring lenses with NA $\sim 0.6-0.7$ and beyond $[24,41,42]$. In D of the Supplemental Material [32], we present how the Heisenberg limit can be reached by increasing the NA of the optical setup up to NA $=1$, and we compare our method to the forward detection scheme adopted for levitated optomechanics [29].

The measurement of the ion position operator $\langle q\rangle$ here presented can be combined with the measurement of $\left\langle q^{2}\right\rangle$ for the analysis of nonclassical states of motion, such as superpositions of Fock states, squeezed states, or cat states, in a full-optical manner. The analysis of quantum states is possible because the relation of $\langle q\rangle$ and $\left\langle q^{2}\right\rangle$ is lower bounded for coherent (classical) states, and any deviation from this law is the signature of a nonclassical state of motion that can be used to characterize the state. Measuring $\langle q\rangle$ and $\left\langle q^{2}\right\rangle$ can be achieved by combining a measurement at the slope of the interference fringes with one at the point of destructive interference in which Eq. (1) expands as $q^{2}$. In the experiments, both $\langle q\rangle$ and $\left\langle q^{2}\right\rangle$ can be measured with similar sensitivity due to the different background count rates. By assuming the fringe contrast of $\mathcal{V} \sim 0.9$ as demonstrated in Ref. [28], we calculate that the relative variation of the count rate induced by a single quantum at the slope is $11 \%$ and at the destructive point is $9 \%$. 
Therefore, by applying the technique described in this Letter at the destructive point of interference, it should be possible to resolve both contributions to $\left\langle q^{2}\right\rangle$ : the dynamic component oscillating at $2 \omega$ and the constant part. This hypothesis is strongly supported by the result reported in Ref. [28] in which, by using the same apparatus, the time average of $\left\langle q^{2}\right\rangle$ was detected with quantum sensitivity by measuring the variation of the interference fringe contrast.

With the presented techniques, all oscillation modes may be analyzed simultaneously. In a trapped ion chain, a chosen ion could be monitored to detect all the frequency modes of the ion string. Thus, our technique may find application for sympathetic cooling and study of oscillations of ion species that cannot be directly laser cooled [43]. Furthermore, the scheme can be used for detecting the motion of ions in a Penning trap by adapting the trap for optical access such as realized in Ref. [44]. These extensions can find applications in fundamental physics beyond the field of quantum optics. Further references used in the Supplemental Material are Refs. [47-51].

We thank P. Bushev, L. Dania, A. Rischka and C. Hempel for insightful discussions and J. Braun for proofreading the manuscript. L. P. and L. S. were supported by Grant No. GA19-14988S of the Czech Science Foundation. This work was supported by the European Commission through Project No. PIEDMONS 801285 and the Marie Skłodowska-Curie Action, Grant No. 801110 (Erwin Schrödinger Quantum Fellowship Program). This work was also supported by the Institut für Quanteninformation $\mathrm{GmbH}$.

Note added in proof.-Since submission of this Letter, feedback cooling of levitated dipolar scatterers has been demonstrated in Ref. [45]. In Ref. [46], it is predicted that our configuration may achieve higher sensitivity to displacements than the technique adopted in Ref. [45]. These findings further strengthen the prospect of achieving ground state cooling of a dipolar scatterer by feedback control with our optical setup.

*Corresponding author. giovanni.cerchiari@uibk.ac.at

[1] S. Eliseev, K. Blaum, M. Block, S. Chenmarev, H. Dorrer, C. E. Düllmann, C. Enss, P. E. Filianin, L. Gastaldo, M. Goncharov et al., Phys. Rev. Lett. 115, 062501 (2015).

[2] Fundamental Physics in Particle Traps, edited by V. M. W. Quint (Springer, New York, 2014), ISBN 978-3662-51173-2, https://doi.org/10.1007/978-3-642-45201-7.

[3] D. Leibfried, D. M. Meekhof, B. E. King, C. Monroe, W. M. Itano, and D. J. Wineland, Phys. Rev. Lett. 77, 4281 (1996).

[4] M. Block, Nucl. Instrum. Methods Phys. Res., Sect. B 376, 265 (2016).
[5] S. Sturm, A. Wagner, B. Schabinger, J. Zatorski, Z. Harman, W. Quint, G. Werth, C. H. Keitel, and K. Blaum, Phys. Rev. Lett. 107, 023002 (2011).

[6] C. Smorra, S. Sellner, M. J. Borchert, J. A. Harrington, T. Higuchi, H. Nagahama, T. Tanaka, A. Mooser, G. Schneider, M. Bohman et al., Nature (London) 550, 371 (2017).

[7] S. Ulmer, C. Smorra, A. Mooser, K. Franke, H. Nagahama, G. Schneider, T. Higuchi, S. Van Gorp, K. Blaum, Y. Matsuda et al., Nature (London) 524, 196 (2015).

[8] M. Niemann, T. Meiners, J. Mielke, M. J. Borchert, J. M. Cornejo, S. Ulmer, and C. Ospelkaus, Meas. Sci. Technol. 31, 035003 (2020).

[9] M. J. Biercuk, H. Uys, J. W. Britton, A. P. VanDevender, and J. J. Bollinger, Nat. Nanotechnol. 5, 646 (2010).

[10] J. D. Jost, J. P. Home, J. M. Amini, D. Hanneke, R. Ozeri, C. Langer, J. J. Bollinger, D. Leibfried, and D. J. Wineland, Nature (London) 459, 683 (2009).

[11] C. Monroe, D. M. Meekhof, B. E. King, and D. J. Wineland, Science 272, 1131 (1996).

[12] D. Leibfried, D. M. Meekhof, C. Monroe, B. E. King, W. M. Itano, and D. J. Wineland, J. Mod. Opt. 44, 2485 (1997).

[13] J. F. Poyatos, R. Walser, J. I. Cirac, P. Zoller, and R. Blatt, Phys. Rev. A 53, R1966 (1996).

[14] D. M. Meekhof, C. Monroe, B. E. King, W. M. Itano, and D. J. Wineland, Phys. Rev. Lett. 76, 1796 (1996).

[15] D. Leibfried, R. Blatt, C. Monroe, and D. Wineland, Rev. Mod. Phys. 75, 281 (2003).

[16] M. Aspelmeyer, P. Meystre, and K. Schwab, Phys. Today 65, No. 7, 29 (2012).

[17] G. Ranjit, M. Cunningham, K. Casey, and A. A. Geraci, Phys. Rev. A 93, 053801 (2016).

[18] A. D. O'Connell, M. Hofheinz, M. Ansmann, R. C. Bialczak, M. Lenander, E. Lucero, M. Neeley, D. Sank, H. Wang, M. Weides et al., Nature (London) 464, 697 (2010).

[19] J. D. Teufel, T. Donner, D. Li, J. W. Harlow, M. Allman, K. Cicak, A. J. Sirois, J. D. Whittaker, K. W. Lehnert, and R. W. Simmonds, Nature (London) 475, 359 (2011).

[20] J. Chan, T. M. Alegre, A. H. Safavi-Naeini, J. T. Hill, A. Krause, S. Gröblacher, M. Aspelmeyer, and O. Painter, Nature (London) 478, 89 (2011).

[21] U. Delić, M. Reisenbauer, D. Grass, N. Kiesel, V. Vuletić, and M. Aspelmeyer, Phys. Rev. Lett. 122, 123602 (2019).

[22] D. Windey, C. Gonzalez-Ballestero, P. Maurer, L. Novotny, O. Romero-Isart, and R. Reimann, Phys. Rev. Lett. 122, 123601 (2019).

[23] U. Delić, M. Reisenbauer, K. Dare, D. Grass, V. Vuletić, N. Kiesel, and M. Aspelmeyer, Science 367, 892 (2020).

[24] F. Tebbenjohanns, M. Frimmer, A. Militaru, V. Jain, and L. Novotny, Phys. Rev. Lett. 122, 223601 (2019).

[25] P. Bushev, D. Rotter, A. Wilson, F. Dubin, C. Becher, J. Eschner, R. Blatt, V. Steixner, P. Rabl, and P. Zoller, Phys. Rev. Lett. 96, 043003 (2006).

[26] P. Bushev, G. Hétet, L. Slodička, D. Rotter, M. A. Wilson, F. Schmidt-Kaler, J. Eschner, and R. Blatt, Phys. Rev. Lett. 110, 133602 (2013).

[27] D. Rotter, M. Mukherjee, F. Dubin, and R. Blatt, New J. Phys. 10, 043011 (2008). 
[28] L. Slodička, G. Hétet, N. Röck, S. Gerber, P. Schindler, M. Kumph, M. Hennrich, and R. Blatt, Phys. Rev. A 85, 043401 (2012).

[29] F. Tebbenjohanns, M. Frimmer, and L. Novotny, Phys. Rev. A 100, 043821 (2019).

[30] G. A. Araneda Machuca, Ph.D. thesis, Universität Innsbruck, 2019.

[31] S. Gerber, D. Rotter, M. Hennrich, R. Blatt, F. Rohde, C. Schuck, M. Almendros, R. Gehr, F. Dubin, and J. Eschner, New J. Phys. 11, 013032 (2009).

[32] See Supplemental Material at http://link.aps.org/ supplemental/10.1103/PhysRevLett.127.063603 for further information regarding the setup and the analysis of the presented experimental results. In particular, we describe the experimental setup of EIT cooling; we discuss the limitations to the interference signal's stability observed during the data acquisition; and we provide an extended description of the physical model and methods adopted for the data analysis.

[33] K. Dholakia, G. Z. K. Horvath, D. M. Segal, R. C. Thompson, D. M. Warrington, and D. C. Wilson, Phys. Rev. A 47, 441 (1993).

[34] M. Schubert, I. Siemers, R. Blatt, W. Neuhauser, and P. E. Toschek, Phys. Rev. A 52, 2994 (1995).

[35] R. Blümel, C. Kappler, W. Quint, and H. Walther, Phys. Rev. A 40, 808 (1989).

[36] The enhancement factor is calculated as the ratio between the two peak heights elevated to the power of $1 / 4$. Furthermore, a reduction factor of $1 / \sqrt{2}$ was multiplied to account for the different orientation of the optical setup and the illuminating beam. The factor assumes no axial micromotion and considers the optical detection setup to have a better overlap along the micromotion direction.
[37] D. Rotter, Ph.D. thesis, Universität Innsbruck, 2008.

[38] S. Sturm, A. Wagner, B. Schabinger, and K. Blaum, Phys. Rev. Lett. 107, 143003 (2011).

[39] S. Eliseev, K. Blaum, M. Block, C. Droese, M. Goncharov, E. Minaya Ramirez, D. A. Nesterenko, Y. N. Novikov, and L. Schweikhard, Phys. Rev. Lett. 110, 082501 (2013).

[40] F. Schmidt-Kaler, J. Eschner, G. Morigi, C. Roos, D. Leibfried, A. Mundt, and R. Blatt, Appl. Phys. B 73, 807 (2001).

[41] G. Araneda, G. Cerchiari, D. B. Higginbottom, P. C. Holz, K. Lakhmanskiy, P. Obšil, Y. Colombe, and R. Blatt, Rev. Sci. Instrum. 91, 113201 (2020).

[42] G. Shu, N. Kurz, M. R. Dietrich, and B. B. Blinov, Phys. Rev. A 81, 042321 (2010).

[43] K. Sheridan and M. Keller, New J. Phys. 13, 123002 (2011).

[44] A. Kellerbauer, A. Fischer, and U. Warring, Phys. Rev. A 89, 043430 (2014).

[45] L. Magrini, P. Rosenzweig, C. Bach, A. DeutschmannOlek, S. G. Hofer, S. Hong, N. Kiesel, A. Kugi, and M. Aspelmeyer, arXiv:2012.15188.

[46] G. Cerchiari, L. Dania, D. S. Bykov, R. Blatt, and T. Northup, arXiv:2103.08322.

[47] R. Lechner, C. Maier, C. Hempel, P. Jurcevic, B. P. Lanyon, T. Monz, M. Brownnutt, R. Blatt, and C. F. Roos, Phys. Rev. A 93, 053401 (2016).

[48] T. Xie, N. Jin, Y. Wang, J. Zhang, M. Um, P. Wang, and K. Kim, J. Opt. Soc. Am. B 36, 243 (2019).

[49] C. Hempel, Ph.D. thesis, Universität Innsbruck, 2014.

[50] Wavelet Analysis and Its Applications, An Introduction to Wavelets, edited by C. K. Chui, Vol. 1 (Academic Press, New York, 1992).

[51] C. Chatfield, The Analysis of Time Series: Theory and Practice (Springer US, New York, 1975), ISBN 978-0-41214180-5, https://doi.org/10.1007/978-1-4899-2925-9. 